\title{
Balkanologie
}

Balkanologie Revue d'études pluridisciplinaires

Vol. XII, $n^{\circ} 1$ | 2010

Volume XII Numéro 1

\section{Kosanić (Zoran), La désagrégation de la fédération yougoslave (1988-1992)}

Paris : L'Harmattan, 2009, 332 p.

\section{Loïc Trégourès}

\section{OpenEdition}

\section{Journals}

Édition électronique

URL : http://journals.openedition.org/balkanologie/2129

DOI : 10.4000/balkanologie.2129

ISSN : 1965-0582

Éditeur

Association française d'études sur les Balkans (Afebalk)

Référence électronique

Loïc Trégourès, « Kosanić (Zoran), La désagrégation de la fédération yougoslave (1988-1992) »,

Balkanologie [En ligne], Vol. XII, n 1 | 2010, mis en ligne le 15 février 2013, consulté le 17 décembre

2020. URL : http://journals.openedition.org/balkanologie/2129 ; DOI : https://doi.org/10.4000/

balkanologie. 2129

Ce document a été généré automatiquement le 17 décembre 2020.

(c) Tous droits réservés 


\title{
Kosanić (Zoran), La désagrégation de la fédération yougoslave (1988-1992)
}

Paris : L'Harmattan, 2009, 332 p.

\author{
Loïc Trégourès
}

\section{RÉFÉRENCE}

Zoran Kosanić, La désagrégation de la fédération yougoslave (1988-1992), Paris : L'Harmattan, $2009,332 \mathrm{p}$.

1 Issu d'une thèse d'histoire contemporaine soutenue en Sorbonne en 2007, l'ouvrage de Zoran Kosanić se présente comme une synthèse essentielle pour comprendre l'ensemble du processus qui a conduit à la disparition de la Yougoslavie au tournant des années 1990. L'auteur insiste en effet dans sa présentation sur le caractère multidimensionnel de son travail de recherche, qui permet de saisir toute la complexité et toutes les connexions existantes entre les différents aspects de la désintégration yougoslave, que ce soit la dimension économique, politique, institutionnelle, militaire, personnelle, ou internationale.

2 Aussi, là où la plupart des travaux de recherche des spécialistes des Balkans occidentaux se sont penchés sur un aspect particulier de la crise yougoslave (la crise économique pour Dijana Pleština ${ }^{1}$, le rôle des personnes pour Christopher Bennett ${ }^{2}$, l'enjeu du Kosovo pour Branka Magaš ${ }^{3}$ et Sabrina Ramet ${ }^{4}$, la responsabilité particulière de la Serbie pour Nebojša Popov ${ }^{5}$, ou encore la mauvaise gestion internationale pour James Gow $^{6}$ ), Kosanić présente l'ambition de les réunir dans une même étude car " chacun de ces segments comporte ses propres ruptures et moments charnières, mais il n'a pu se construire qu'au travers des autres et seule une approche multidimensionnelle permet d'embrasser le sujet dans toute sa complexité» (p. 10).

3 L'auteur va donc revenir avec précision et minutie sur tous les faits qui ont conduit, de 1988 à 1992, à l'implosion yougoslave et à la guerre. Le choix de commencer son étude en 1988 est en soi sujet à débat. Kosanić le justifie par le fait que 1988 correspond au 
déclenchement de la "révolution anti-bureaucratique" en Serbie, et que c'est «l'année où les fractures, désormais ouvertes entre les Républiques et les Provinces autonomes, s'étalent au grand jour » (p. 10). Ce faisant, il refuse de dater le début de l'implosion yougoslave à 1980 comme beaucoup l'ont fait. Valider ce choix reviendrait en effet d'une certaine façon à acter que la Yougoslavie n'était pas viable sans la clé de voûte qu'était Tito, ce qui exonérerait de leurs responsabilités les dirigeants des différentes républiques qui ont conduit le pays à la dislocation et à la guerre. Commencer en 1988 permet au contraire de refuser le déterminisme historique, de mettre en lumière les actes de toutes les républiques et de leurs dirigeants, et d'en tirer les conclusions quant aux responsabilités des uns et des autres dans la désagrégation yougoslave.

4 Comme l'immense majorité des auteurs, Kosanić souscrit à l'idée selon laquelle Slobodan Milošević est le principal fossoyeur de la Yougoslavie. Il introduit toutefois deux autres éléments importants, à savoir la responsabilité de " pouvoirs analogues de transformation radicale du système fédéral et social (qui) ont appartenu aux autres dirigeants républicains, notamment ceux des républiques à l'origine de la création de la première Yougoslavie, à savoir la Slovénie et la Croatie » (p. 11), ainsi que celle de la Communauté Européenne, novice sur le scène internationale et dont les contradictions, les tergiversations et les erreurs de jugement auront alimenté l'inextricable de la crise yougoslave à partir de 1991.

5 L'ouvrage se découpe en trois parties. La première partie revient sur les problèmes structurels de la Yougoslavie (crise économique, contradictions de la Constitution de 1974), ainsi que sur les premiers signes d'hostilité entre les républiques. Kosanić consacre notamment un chapitre à la prise de pouvoir de Slobodan Milošević en Serbie en 1987, ainsi qu'à la question du Kosovo, enjeu crucial puisque c'est sur lui que se cristallisera la rupture slovéno-serbe. Le dernier chapitre de cette partie est une brillante analyse de la façon dont les républiques (en particulier la Serbie et la Croatie) ont pris possession des médias et en ont fait un instrument de propagande nationaliste par le déversement de fausses informations et par une rhétorique haineuse envers les autres nationalités. À cela s'ajoute un retour sur la victoire culturelle des programmes nationaux en Serbie, grâce au mémorandum de la SANU, en Croatie à travers le "néomaspok", ainsi qu'en Slovénie après la publication d'un Programme national slovène dans la Nova Revija.

6 Ainsi sont posés les jalons culturels, économiques et institutionnels qui participent du repli sur soi de chaque république et qui mèneront aux deux coups de grâce de l'année 1990 , à savoir le retrait prémédité de la délégation slovène du $14^{\text {ème }}$ Congrès extraordinaire de la LCY, et l'organisation des premières élections démocratiques non pas à l'échelle fédérale, mais à l'échelle des républiques ce qui ôte toute légitimité aux institutions fédérales et ses dirigeants, Ante Marković en tête.

7 La deuxième partie se concentre sur les aspects politiques et institutionnels qui ont conduit la Yougoslavie de la dislocation à la guerre entre 1990 et 1991. L'auteur revient d'abord longuement sur ce fameux $14^{\text {ème }}$ Congrès de la LCY, puis sur les élections de 1990 dans chaque République, présentant les forces en présence et les conséquences des résultats pour les républiques et pour la fédération.

8 Il analyse dans un second temps les trois points de fixation de la question yougoslave, ceux par qui la désagrégation et la guerre sont arrivées. Le premier d'entre eux est le processus politique et institutionnel de séparation de la république de Slovénie depuis la publication par la LC de Slovénie du « Document fondamental de la Slovénie » le 27 
juin 1989, document qui stipule que «la nation slovène souhaite vivre dans un État souverain du peuple slovène» (p. 159), jusqu'aux amendements approuvés par le parlement de Slovénie en septembre 1989 qui inversent la hiérarchie des normes entre le droit fédéral et le droit des républiques, ce qui est un premier pas juridique vers une remise en question fondamentale de la Yougoslavie.

Le second foyer de tension est le Kosovo dans lequel la Serbie de Milošević a repris les choses en main depuis 1989 par une série d'amendements qui réduisent à néant l'autonomie de la province. Les Albanais répondent par la proclamation clandestine de l'indépendance de la république du Kosovo et par l'établissement d'institutions parallèles ${ }^{7}$, ainsi que d'un boycott de la vie politique serbe.

10 Enfin, le troisième point concerne la Croatie et la tension toujours plus vive qui s'installe entre d'une part le HDZ de Franjo Tuđman, et d'autre part le SDS, qui débouche sur une insurrection séparatiste serbe en Krajina et la création d'une entité autonome, la Republika Srpska Krajina (RSK) en décembre 1991 avec Milan Babić comme premier président.

11 Cette situation insurrectionnelle permet à l'auteur de revenir en détail sur le rôle et l'évolution de l'armée yougoslave (JNA) pendant la crise, qui va progressivement se transformer en bras armé de la politique de Milošević, a fortiori après que le président serbe de la fédération, Borisav Jović annonce le retrait piteux de la JNA de Slovénie ${ }^{8}$, accréditant ainsi la thèse d'un accord entre les Slovènes et Milošević sur leur indépendance. Dès lors, alors que la Macédoine demande le retrait des troupes fédérales de son territoire en mars 1991 et que les sabotages et les désertions se multiplient en son sein, « le mois de mars 1991 constitue une rupture définitive dans les relations entre la présidence fédérale et les républiques. Le rapport de force qui s'établit entre les différentes républiques met définitivement hors-jeu les instances fédérales dans leurs différentes composantes, incluant aussi la JNA » (p. 208).

Dans la troisième et dernière partie, Zoran Kosanić introduit le facteur international dans l'équation yougoslave. En effet, à partir du second semestre 1991, la Communauté Européenne va s'investir dans le dossier yougoslave, désireuse de montrer son unité et sa puissance dans un monde qui s'apprête à devenir multipolaire. C'est sous l'égide de la CE que les accords de Brioni seront signés en juillet 1991. Ceux-ci entérinent la défaite de la JNA en Slovénie sans pour autant statuer sur le degré de souveraineté de celle-ci. Dans le même temps, ils consacrent l'internationalisation de la question yougoslave et une implication toujours plus forte de la Communauté Européenne.

L'analyse que fait l'auteur de l'action diplomatique de la CE et de chacun de ses membres le conduit à deux conclusions. D'une part, la Communauté Européenne se révèle être une coquille vide dont les divergences en son sein sont grandes, elle est maladroite, estime mal la situation sur le terrain, et ses moyens d'actions sont très limités ce qui la conduit à l'impuissance, et la contraint à faire appel à l'ONU et aux États-Unis. D'autre part, Kosanić revient en détail sur le cycle de conférences de La Haye présidées par Lord Carrington, et le rôle joué par la Commission d'arbitrage, présidée par Robert Badinter. D'après l'auteur, cette commission, qui a paré d'habits juridiques les indépendances des différentes républiques issues de la Yougoslavie, «a péché du fait de la primauté évidente du jeu politique sur les considérations strictement juridiques» (p. 317). Habilement, celle-ci a en effet conclu à la « dissolution » de la Yougoslavie en évitant de parler de processus de "sécession" ce qui 
permettait à l'Europe de nier toute ingérence dans les affaires d'un État sujet de droit international.

En outre, Kosanić met en évidence des contradictions entre ses différents avis, par exemple sur le statut du peuple serbe, décrit comme une minorité en BosnieHerzégovine où il est un des trois peuples constituants (pp. 308-309), ainsi que son manque de discernement lorsqu'elle conditionne la reconnaissance de l'indépendance de la Bosnie-Herzégovine à l'organisation d'un référendum avec pour règle 1 homme = 1 voix dans un pays où la clé nationale avait toujours été la règle sous Tito.

Dans sa conclusion, Zoran Kosanić s'interroge sur un possible affaissement de la souveraineté politique compte tenu de l'émergence sur la scène internationale de nouveaux acteurs, comme les organisations humanitaires qui ont poussé à l'intervention de l'OTAN en 1999 en Serbie et au Kosovo. Cette intervention, dont l'auteur ne discute pas l'éventuelle légitimité, n'en reste pas moins illégale au point de vue du droit international. Dès lors, l'indépendance du Kosovo s'opposant aux conclusions de la Commission Badinter qui avait elle-même interprété à sa guise le droit international pour légitimer les indépendances des républiques yougoslaves, la remise en cause des normes juridiques internationales élémentaires, en particulier la Charte des Nations Unies ne risque-t-elle pas de conduire à un dangereux relativisme de la part des grandes puissances dans l'approche des questions internationales?

On peut faire trois types de critiques au travail de Zoran Kosanić. Tout d'abord, par son pari de produire un ouvrage multidimensionnel, l'auteur en oublie de discuter les nécessaires débats sur les multiples causes de la désagrégation yougoslave, les importances différentes que les unes et les autres ont pu avoir, et se contente d'une histoire factuelle où l'on se perd parfois entre les dates, les faits, les acronymes de partis et les résultats d'élections. Or, il est des auteurs, comme Andrew Watchel par exemple, qui estiment que le facteur culturel et éducatif a été bien plus capital dans la dislocation yougoslave que les problèmes politiques, économiques et institutionnels qu'a connus le pays. Watchel écrit notamment :

L'abandon de la tentative de création d'une nation culturelle de la part des élites politiques et culturelles a créé les conditions de la désagrégation de l'État yougoslave. Cela ne veut pas dire que l'analyse culturelle à elle seule permet d'expliquer l'implosion de la Yougoslavie. Mais la crise politique et économique que le pays traversait dans les années 80 , aussi réelle soit-elle, n'aurait jamais mené à la disparition du pays si une solide éducation culturelle de la nation yougoslave avait été inculquée à tous9.

17 Il eût été intéressant que l'auteur se positionne par rapport à ce type de débat.

18 La deuxième critique porte sur le choix de l'auteur de présenter la dislocation de la Yougoslavie comme un processus qui s'est essentiellement effectué "par le haut". Or, et Watchell a donné un élément de réponse, si ce sont les élites politiques et intellectuelles qui ont bel et bien mis le feu aux poudres, ce sont des gens "normaux" qui ont été convaincus par les discours de haine des médias, ce sont des gens "normaux" qui se sont engagés pour faire la guerre et pour commettre des atrocités, parfois contre leurs propres voisins. Ainsi, il manque sans aucun doute des références et des explications d'ordre psychologique, sociologique et anthropologique afin de réellement cerner toute la complexité de la dislocation yougoslave dans la guerre, qu'on ne peut réduire à des amendements constitutionnels, des déclarations d'indépendance et des négociations entre dirigeants, aussi essentiels ces éléments 
formels soient-ils. La désagrégation de la Yougoslavie s'est également faite "par le bas » et cet aspect est absent du travail de l'auteur.

Enfin, si l'auteur fait preuve de rigueur dans le référencement de ses sources, la grande majorité en langue locale, tout ce qui est dit est relativement connu ${ }^{10}$ et $n^{\prime}$ 'offre pas vraiment de renouveau au niveau des débats historiques ou historiographiques.

Malgré ces écueils, parfois difficilement évitables en raison des choix de départ de l'auteur, La désagrégation de la fédération yougoslave représente un essentiel travail de synthèse très fouillé et rigoureux, absolument nécessaire dans un univers littéraire où les livres sur la Yougoslavie sont extrêmement nombreux et se focalisent souvent sur une dimension précise du conflit, ou bien sur une république particulière ${ }^{11}$.

\section{NOTES}

1. Pleština (Dijana), Regional Development in Communist Yugoslavia: Success, Failure and Consequences, Boulder : Westview Press, 2002.

2. Bennett (Christopher), Yugoslavia's Bloody Collapse: Causes, Course and Consequences, New York: New York University Press, 1995.

3. Magaš (Branka), Žanić (Ivo) eds, The War in Croatia and Bosnia-Herzegovina 1991-1995, London : Frank Cass, 2001.

4. Ramet (Sabrina), Nationalism and federalism in Yugoslavia 1962-1991, Indianapolis, 1992 ; Balkan Babel. From the Death of Tito to the Fall of Milošević, Boulder : Westview, 2002 (4 éd.).

5. Popov (Nebojša) dir., Radiographie d'un nationalisme, les racines serbes du conflit yougoslave, Paris : Les Éditions de l'Atelier, 1998.

6. Gow (James), Triumph of the Lack of Will : International Diplomacy and the Yugoslav War, New York : Columbia University Press, 1997.

7. Sur ces institutions parallèles, voir l'ouvrage essentiel de Kostovičová (Denisa), The Kosovo, the Politics of Identity and Space, Londres : Routledge, 2005, étonnamment négligé par l'auteur.

8. Jović (Borisav), Poslednji dani SFRJ, izvodi iz dnevnika [Les derniers jours de la RSFY], Belgrade : Politika, 1995 ; Repe (Božo), Viri o demokratizaciji in osamosvojitvi Slovenije [Les origines de la démocratisation et de l'indépendance de la Slovénie], Ljubljana: Narodna in univerzitetna knižnica, 2002.

9. Watchel (Andrew), Making a Nation, Breaking a Nation: Literature and Cultural Politics in Yugoslavia, Stanford : Stanford University Press, 1998, p. 184.

10. On peut se référer par exemple au film « The Death of Yugoslavia », paru en serbo-croate sous le titre « Smrt Jugoslavije ", écrit par Silber (Laura) et Little (Allan), documentaire découpé en 6 parties contenant des documents vidéos inédits et les témoignages des principaux dirigeants de l'ancienne Yougoslavie, produit et diffusé par la BBC le 3 septembre 1995.

11. Par exemple, la remarquable étude de Bougarel (Xavier), Bosnie, anatomie d'un conflit, Paris : Éditions de La Découverte, 1996. 


\section{AUTEURS}

\section{LOÏC TRÉGOURÈS}

Diplômé de l'IEP de Lille, doctorant en sciences politiques à l'Université Libre de Bruxelles loictregoures@yahoo.fr 